\title{
Preference of Dogs between Two Commercially Available Oral Formulations of Ectoparasiticide Containing Isoxazolines, Afoxolaner or Fluralaner
}

\author{
Lénaïg Halos ${ }^{1}$, Douglas S. Carithers ${ }^{2}$, Ruchika Solanki², Heather Stanford ${ }^{3}$, Sheila J. Gross ${ }^{4}$ \\ ${ }^{1}$ Merial S.A.S., Lyon, France \\ ${ }^{2}$ Merial Limited, Duluth, USA \\ ${ }^{3}$ Summit Ridge Farms, Susquehanna, USA \\ ${ }^{4} 26$ Bayberry Close, Piscataway, USA \\ Email:
}

Received 27 January 2015; accepted 11 February 2015; published 11 February 2015

Copyright (C) 2015 by authors and Scientific Research Publishing Inc.

This work is licensed under the Creative Commons Attribution International License (CC BY). http://creativecommons.org/licenses/by/4.0/

c. (i) Open Access

\section{Abstract}

Because of their convenience, oral dosage forms are an emerging trend in companion animal formulations. Ectoparasiticides represent a significant proportion of the treatments administered to pets, and recently oral formulations have been commercialized. They have to demonstrate high palatability to ensure that they are voluntarily accepted by the animal especially because they are repeatedly offered medications. The present study aimed to compare the dog's preference between two commercially available oral ectoparasiticide formulations containing either afoxolaner (NexGard $^{\circledR}$, Merial) or fluralaner (Bravecto ${ }^{\circledR}$, MSD Animal Health). In two separate experiments, 225 individual dogs (115 and 110 respectively) were offered a choice of afoxolaner or fluralaner chewable tablets. The 225 dogs were given an opportunity to smell both products, and then the products were simultaneously offered to each dog by hand for 4 consecutive days with products offered from alternate hands on each day. Individual consumption and related behaviors were recorded. The same individual offered the products to the dogs throughout each study. The total number of chewable tablets for each formulation was recorded and preference was evaluated as the consumption of a given formulation during more days. A total amount of 797 tablets were consumed by the 225 dogs during the 4 days of the studies. A total of 573 (71.9\%) afoxolaner chews and $224(28.1 \%)$ fluralaner chews were consumed voluntarily. The overall consumption ratio was 2.56 to 1 for NexGard $^{\text {TM }}$ to Bravecto ${ }^{\circledR}$, with significantly $(p<0.0001)$ more dogs consuming NexGard ${ }^{\circledR}$ than Bravecto $^{\circledR}$ on each day. As for dogs demonstrating a preference over the entire test period; $83 \%$ of the dogs tested preferred NexGard ${ }^{\circledR}$ to Bravecto ${ }^{\circledR}$, resulting in a preference ratio of 5 to 1 for afox-

\footnotetext{
"Corresponding author.
}

How to cite this paper: Halos, L., Carithers, D.S., Solanki, R., Stanford, H. and Gross, S.J. (2015) Preference of Dogs between Two Commercially Available Oral Formulations of Ectoparasiticide Containing Isoxazolines, Afoxolaner or Fluralaner. Open Journal of Veterinary Medicine, 5, 25-29. http://dx.doi.org/10.4236/ojvm.2015.52004 
olaner formulation versus fluralaner formulation. This study demonstrated that when dogs were offered a choice between the two ectoparasiticide products, a significant preference for NexGard ${ }^{\circledR}$ was observed.

\section{Keywords}

\section{Palatability, Preference, Oral Ectoparasiticide Treatment, Compliance}

\section{Introduction}

Because regular treatment of companion animals by their owners is generally easier said than done, the development of formulations allowing maximization of compliance and convenience has become a major trend of drug development [1]. Oral formulations offer an easy fit with this need, if they are highly palatable [2]. Regarding veterinary medicine, consideration of palatability has long been limited to discussions associated with pet food. Now, as more oral pharmaceutical formulations are being developed for regular use in healthy companion animals, efforts are being made to ensure that these formulations are voluntarily accepted by companion animals. If the product is highly palatable, it will be considered a special treat by both the pet and the owner, preserving the human-animal bond. Highly palatable formulations offer thus great advantages for repeatedly administered drug formulations, such as ectoparasiticide treatments.

There is no standard, widely accepted definition of palatability. The following definition has been proposed: "the term 'palatability' refers to the voluntary (free choice) acceptance or ingestion of a pharmaceutical composition by companion animals, as measured by a standard palatability test, such as an acceptance, preference or consumption test" [2]. Recently, the Committee for Medicinal Products for Veterinary Use (CVMP) of the European Medical Agency drafted a Guideline on the demonstration of palatability of veterinary medicinal products. In this guideline, palatability is defined as the property of being acceptable to the mouth, "pleasant to the taste" or "acceptable to the taste". When applied to a VMP, this term suggests that the product is palatable enough to ensure voluntary uptake [3]. Determining palatability in dogs and cats is complicated due to the subjective nature of the individual animal's response at any one time or over a period of time. A preference test is a two-option free choice testing format, designed to address the question: "does the animal prefer one option to another". Since there is an alternative, the animals can exercise a choice, such tests are more sensitive than a simple acceptance test [1].

Advances in research for ectoparasitological control have brought new therapeutic drugs to the market for clinical use [4]. Recently, two compounds from a new structurally-unique isoxazoline class which acts as a novel and specific blocker of insect ligand-gated chloride ion channels have been commercialized for ectoparasiticide treatment of dogs: afoxolaner [5], formulated in a soft chewable tablet with artificial beef flavoring (NexGard ${ }^{\circledR}$ Merial) [6] and fluralaner [7] formulated as a pork liver-flavored chewable tablet (Bravecto ${ }^{\circledR}$ MSD Animal Health) [8]. Those two products are the first oral ectoparasiticides soft chewable tablets for dogs in the market. The palatability of NexGard ${ }^{\circledR}$ has been demonstrated very high in an acceptance test conducted on 30 dogs for the registration of the product and demonstrating an overall voluntary acceptance rate of 90\% [9].

The goal of the present studies was to examine the preference exhibited by dogs when simultaneously offering a choice of both oral formulations, allowing a calculation of a preference ratio.

\section{Materials and Methods}

\subsection{Test Animals}

Two separate experiments of similar design were conducted, the only difference being the number of dogs per experiment. A total of 225 individual dogs (115 and 110 respectively) were included. The dogs were intact male (98) and female (127) purpose-bred beagle dogs, ranging from 10 months to 12.6 years of age and weighing between 6.49 to $21.69 \mathrm{~kg}(14.29-47.78 \mathrm{lbs})$ at study initiation in the Summit Ridge Farms colony (USA).

\subsection{Animal Management}

The studies were conducted by an experienced and independent contract research organization. Animals were 
managed similarly and with due regard for their well-being. Animals were handled in compliance with the Merial's Institutional Animal Care and Use Committee (IACUC) approvals which follow EU Directive 2010/63/EU for animal experiments, by the Summit Ridge Farms' IACUC, and was in compliance with the Animal Welfare Act (USA). The dogs were allowed to acclimate to the test facility for at least 7 days before the start. Willingness of the dogs to accept treats from an open hand was determined prior to study initiation. All dogs were fed each morning, evaluated twice daily, and fresh tap water was available by means of an automatic watering system. The dogs were offered their normal rations, which were supplied in appropriate amounts according to body weight. Cages and food bowls were cleaned daily and sanitized. Dogs were maintained with a 12-hour-light/12hour-dark cycle with every attempt made to keep temperature ranges within targeted conditions (from $10^{\circ} \mathrm{C}$ to $\left.30^{\circ} \mathrm{C}\right)$.

\subsection{Treatment}

The two experiments were conducted on four consecutive days. The entire group of dogs was tested in the same manner by the same investigator on each day. Prior to the first treatment on Day 0 , the test articles were randomly assigned as A (NexGard ${ }^{\circledR}$ chewable tablets for dogs $>4-10 \mathrm{~kg}(28.3 \mathrm{mg}$ of afoxolaner) and B (Bravecto $^{\circledR}$ chewable tablets for very small dogs $(2-4.5 \mathrm{~kg})(112.5 \mathrm{mg}$ of fluralaner). The dosages were selected to minimize the dose of medication administered to dogs during the study and to make the 2 products closer in size.

\subsection{Offering Procedure}

On Day 0, tablet A was offered in the left hand and tablet B was offered in the right hand. The hands holding the products were reversed on each day of the study to minimize any tendency for "handed-ness".

The same personnel conducted the offering and recording procedures for each dog and offering began four hours after the regular morning feeding. At each offering the products were held in the fingertips, and dogs were allowed to sniff each product. Then both hands were withdrawn, then the products moved to the palms, then the opened hands were positioned at the level of the dog's head approximately one foot apart and equidistant from the dog, for one minute, or until the dog took a tablet from one hand.

If the dog did not take either product within one minute, "none" was recorded for that dog on that particular day.

If a product was taken from one hand, the other hand was closed around the remaining product and the technician placed both hands behind his or her back. The dog was observed for consumption of the product. In the event that the dog dropped the product, a portable resting board was placed in each dog's enclosure in order to prevent the product from falling through, onto the floor. If all or part of the product was expelled from the dog's mouth, the dog was allowed approximately half a minute to take the product back in his/her mouth and consume it. After 30 seconds, if the dog did not take the product which was chosen first, back into its mouth, the second product was offered again.

The product consumed in its entirety was the preferred product on that day, and those data were recorded so each dog's overall preference could be determined. Observations were made as to whether some or the entire product was dropped from the mouth.

\subsection{Statistical Methods}

An overall consumption ratio was calculated based on the total numbers of tablets of each product consumed during the study. Proportion of tablets of each product consumed was compared using a chi squared test.

To determine the overall preference for Product A or Product B, the number of times each product was consumed was compared for each dog:

1) If one product was consumed more frequently than the other, the dog was defined as preferring that product;

2) If the number of items consumed was equal $(0 / 0,1 / 1,2 / 2)$, the dog demonstrated no preference, so it was defined as "neither".

The preference, based on complete consumption, was scored daily for each dog and the proportion of dogs preferring one or the other product over the four daily offerings was compared to $50 \%$ using a chi squared test. The 50:50 ratio corresponds to the count that would have been expected if there were no difference in preference between the two formulations, i.e., both products chosen equally. Dogs that consumed neither product (daily) or that preferred neither product (overall) were excluded for the calculation of the preference ratio. 


\section{Results}

A total amount of 797 tablets were consumed by the 225 dogs during the 4 days of the studies. A total of 573 (71.9\%) afoxolaner chews and 224 (28.1\%) fluralaner chews were consumed. It gives a 2.56 to 1 overall consumption ratio in favor of NexGard ${ }^{\circledR}$ over Bravecto ${ }^{\circledR}$ (Table 1).

On each study day, of those dogs consuming any product, significantly $(\mathrm{p}<0.01)$ more dogs consumed Nex$\operatorname{Gard}^{\circledR}$ than Bravecto ${ }^{\circledR}$, compared to equal consumption (50\%), with NexGard ${ }^{\circledR}$ consumption at $81 \%, 71 \%, 73 \%$ and $63 \%$.

Overall 4 days, NexGard ${ }^{\circledR}$ was preferred (chosen and completely consumed) by 143 dogs, whereas Bravecto ${ }^{\circledR}$ was preferred by 29 dogs. Fifty-three dogs displayed no preference. For those dogs that showed a preference, significantly $(\mathrm{p}<0.01)$ more dogs preferred NexGard ${ }^{\circledR}$ over Bravecto ${ }^{\circledR}(83 \%$ vs. $17 \%)$, resulting in a preference ratio of 5 to 1 for afoxolaner formulation.

\section{Discussion}

The present study demonstrated an overall significant preference for the commercially available soft chew formulation of afoxolaner versus the commercially available formulation of fluralaner. The experiments were conducted twice on a large number of subjects in order to overcome variability biaises [10]. No specific tests have been performed for palatability comparisons of the isoxazoline compounds, themselves. The overall palatability of both products is thought to be linked to their general composition. The three main ingredients in the Nex$\operatorname{Gard}^{\circledR}$ formulation are corn starch, soy protein fines, and beef braised flavouring [6], whereas the 3 main ingredients in the Bravecto ${ }^{\circledR}$ chewable tablets are pork liver flavor, sucrose, and corn starch [8]. The natural preference of dogs is thought to trend towards meat-based flavors and complex mixtures of flavors [2]. No specific characteristic of the primary ingredients in each formulation can explain the significant difference in the desirability of the products. It is known that, in addition to the ingredients present in a given formulation, the manufacturing processes can also have an impact on palatability. This may explain the difference observed in the present study.

Ectoparasitoses in carnivores account for the most common afflictions of pets [11] [12]. As an example, flea infestation, with a prevalence of 5\% - 10\% in Europe among the canine and feline population, is the most common ectoparasitosis. Ticks are also common, and are more dangerous given their role as vector of numerous diseases, including zoonotic agents. Pet owners recognize the need to protect their companions from fleas, but it has been demonstrated that one of the main reasons for pet owner-related flea-control failure is the lack of compliance [13] [14]. Thus, any means that would increase the convenience of administration should also favor compliance, increasing overall prevention against ectoparasites. In addition to the primary attributes of a pharmaceutical product, efficacy and safety, palatability is a desire attribute because it affects convenience and compliance [2].

\section{Conclusion}

This study demonstrated that when dogs are offered a choice between the two recent commercially available formulations of isoxazoline compounds, afoxolaner formulated in a soft, beef-flavored chewable tablet (Nex$\left.\operatorname{Gard}^{\circledR}\right)$ and fluralaner formulated as a chewable tablet $\left(\right.$ Bravecto $\left.^{\circledR}\right)$, a significant preference for the afoxolaner formulation was observed.

Table 1. Overall daily counts of chewable tablets that have been swallowed.

\begin{tabular}{cccc}
\hline & \multicolumn{3}{c}{ Daily consumed (completely ingested) counts } \\
\hline Day & Tablet A (afoxolaner) & Tablet B (fluralaner) & p-value of Chi $^{2}$ square \\
\hline 0 & $152(80.9 \%)$ & $36(19.1 \%)$ & $<0.0001$ \\
1 & $139(70.9 \%)$ & $57(29.1 \%)$ & $<0.0001$ \\
2 & $151(73.3 \%)$ & $55(26.7 \%)$ & $<0.0001$ \\
3 & $131(63.3 \%)$ & $76(36.7 \%)$ & $<0.0001$ \\
\hline
\end{tabular}




\section{Conflict of Interest}

Merial S.A.S. funded this study. The authors are either employees or contractors of Merial. NexGard ${ }^{\circledR}$ is a trademark of Merial Limited.

Bravecto $^{\circledR}$ is a registered trademark of Intervet Inc., a subsidiary of Merck and Company (d/b/a Merck Animal Health/MSD Animal Health).

\section{References}

[1] Petry, G., Fourie, J. and Wolken, S. (2014) Comparison of the Palatability of a New Flavoured Drontal ${ }^{\circledR}$ Plus Tablet (Drontal ${ }^{\circledR}$ Plus Treat $10 \mathrm{~kg}$ ) and Milbemax ${ }^{\circledR}$ Chewable Tablets When Presented to Privately Owned Dogs. Open Journal of Veterinary Medicine, 4, 163-169. http://dx.doi.org/10.4236/ojvm.2014.48018

[2] Thombre, A.G. (2004) Oral Delivery of Medications to Companion Animals: Palatability Considerations. Advanced Drug Delivery Reviews, 56, 1399-1413. http://dx.doi.org/10.1016/j.addr.2004.02.012

[3] Committee for Medicinal Products for Veterinary Use (CVMP) of the European Medicines Agency (2014) Guideline on the Demonstration of Palatability of Veterinary Medicinal Products. http://www.ema.europa.eu/docs/en_GB/document_library/Scientific_guideline/2012/11/WC500134876.pdf

[4] Beugnet, F. and Franc, M. (2012) Insecticide and Acaricide Molecules and/or Combinations to Prevent Pet Infestation by Ectoparasites. Trends in Parasitology, 28, 267-279 http://dx.doi.org/10.1016/i.pt.2012.04.004

[5] Shoop, W.L., Hartline, E.J., Gould, B.R., Waddell, M.E., McDowell, R.G., Kinney, J.B., Lahm, G.P., Long, J.K., Xu, M., Wagerle, T., Jones, G.S., Dietrich, R.F., Cordova, D., Schroeder, M.E., Rhoades, D.F., Benner, E.A. and Confalone, P.N. (2014) Discovery and Mode of Action of Afoxolaner, a New Isoxazoline Parasiticide for Dogs. Veterinary Parasitology, 201, 179-189. http://dx.doi.org/10.1016/j.vetpar.2014.02.020

[6] European Medicines Agency (2014) Nexgard Summary of Product Characteristics. http://www.ema.europa.eu/docs/en_GB/document_library/EPAR_-_Product_Information/veterinary/002729/WC50016 4067.pdf

[7] Walther, F.M., Allan, M.J., Roepke, R.K. and Nuernberger, M.C. (2014) Safety of Fluralaner Chewable Tablets (Bravecto), a Novel Systemic Antiparasitic Drug, in Dogs after Oral Administration. Parasites and Vectors, 7, 481.

[8] European Medicines Agency (2014) Bravecto Summary of Product Characteristics. http://www.ema.europa.eu/docs/en_GB/document_library/EPAR _ Product Information/veterinary/002526/WC50016 3859.pdf

[9] European Medicines Agency (2014) NexGard Chewable Tablets for Dogs. Registration File for Marketing Authorisation. (EU/2/13/159) Market Authorization granted on 11.2.2014.

[10] Araujo, J.A., Studzinski, C.M., Larson, B.T. and Milgram, N.W. (2004) Comparison of the Cognitive Palatability Assessment Protocol and the Two-Pan Test for Use in Assessing Palatability of Two Similar Foods in Dogs. American Journal of Veterinary Research, 65, 1490-1496. http://dx.doi.org/10.2460/ajvr.2004.65.1490

[11] Guaguère, E. and Beugnet, F. (2008) Parasitic Skin Conditions. In: Guaguère, E., Prélaud, P. and Craig, M., Eds, A Practical Guide to Canine Dermatology, Kalianxis, Paris, 179-226.

[12] Berrada, Z.L. and Telford III, S.R. (2009) Burden of Tick-Borne Infections on American Companion Animals. Topics in Companion Animal Medicine, 24, 175-181. http://dx.doi.org/10.1053/j.tcam.2009.06.005

[13] Coles, T.B. and Dryden, M.W. (2014) Insecticide/Acaricide Resistance in Fleas and Ticks Infesting Dogs and Cats. Parasites and Vectors, 7, 8. http://dx.doi.org/10.1186/1756-3305-7-8

[14] Halos, L., Beugnet, F., Cardoso, L., Farkas, R., Franc, M., Guillot, J., Pfister, K. and Wall, R. (2014) Flea Control Failure? Myths and Realities. Trends in Parasitology, 30, 228-233. http://dx.doi.org/10.1016/j.pt.2014.02.007 
Scientific Research Publishing (SCIRP) is one of the largest Open Access journal publishers. It is currently publishing more than 200 open access, online, peer-reviewed journals covering a wide range of academic disciplines. SCIRP serves the worldwide academic communities and contributes to the progress and application of science with its publication.

Other selected journals from SCIRP are listed as below. Submit your manuscript to us via either submit@scirp.org or Online Submission Portal.
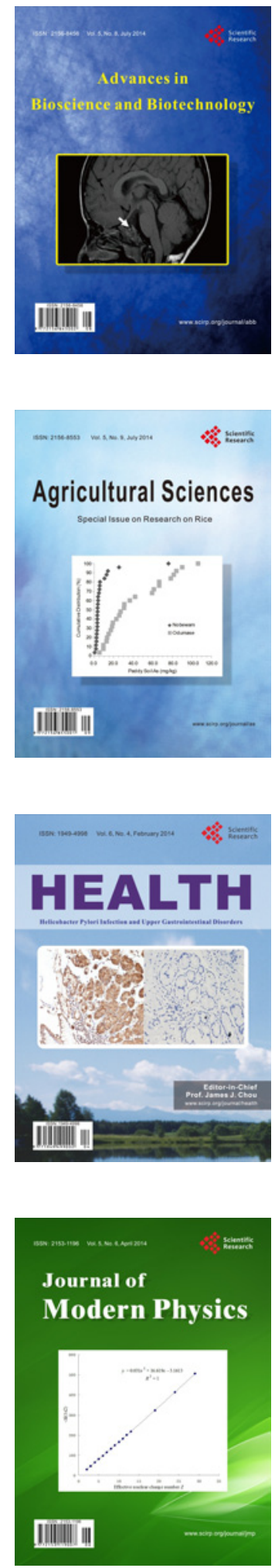
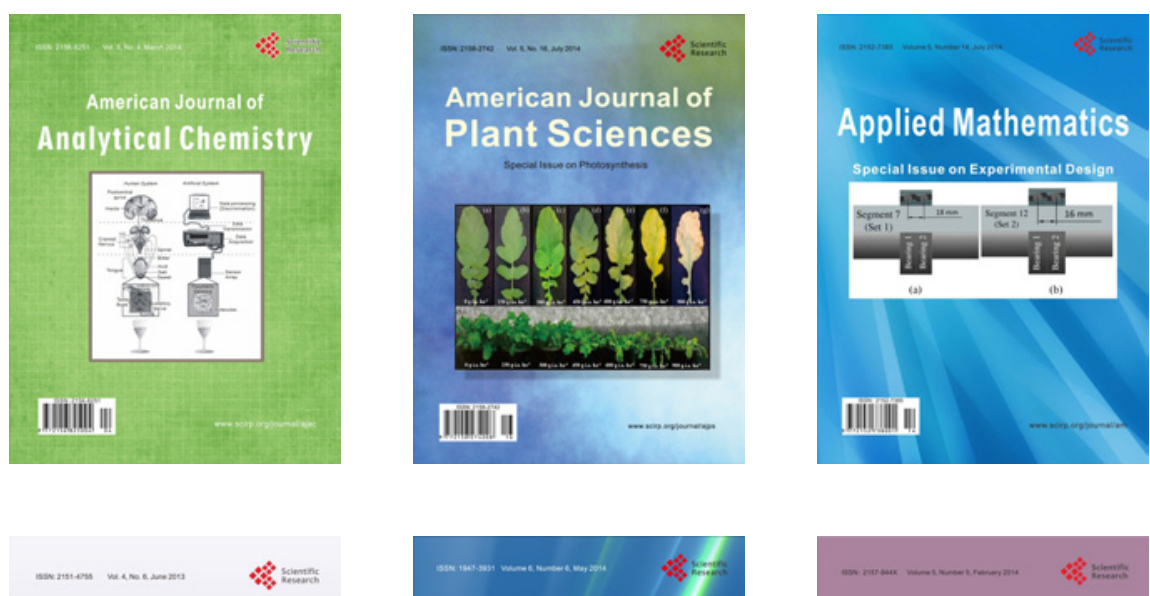

Creative Education
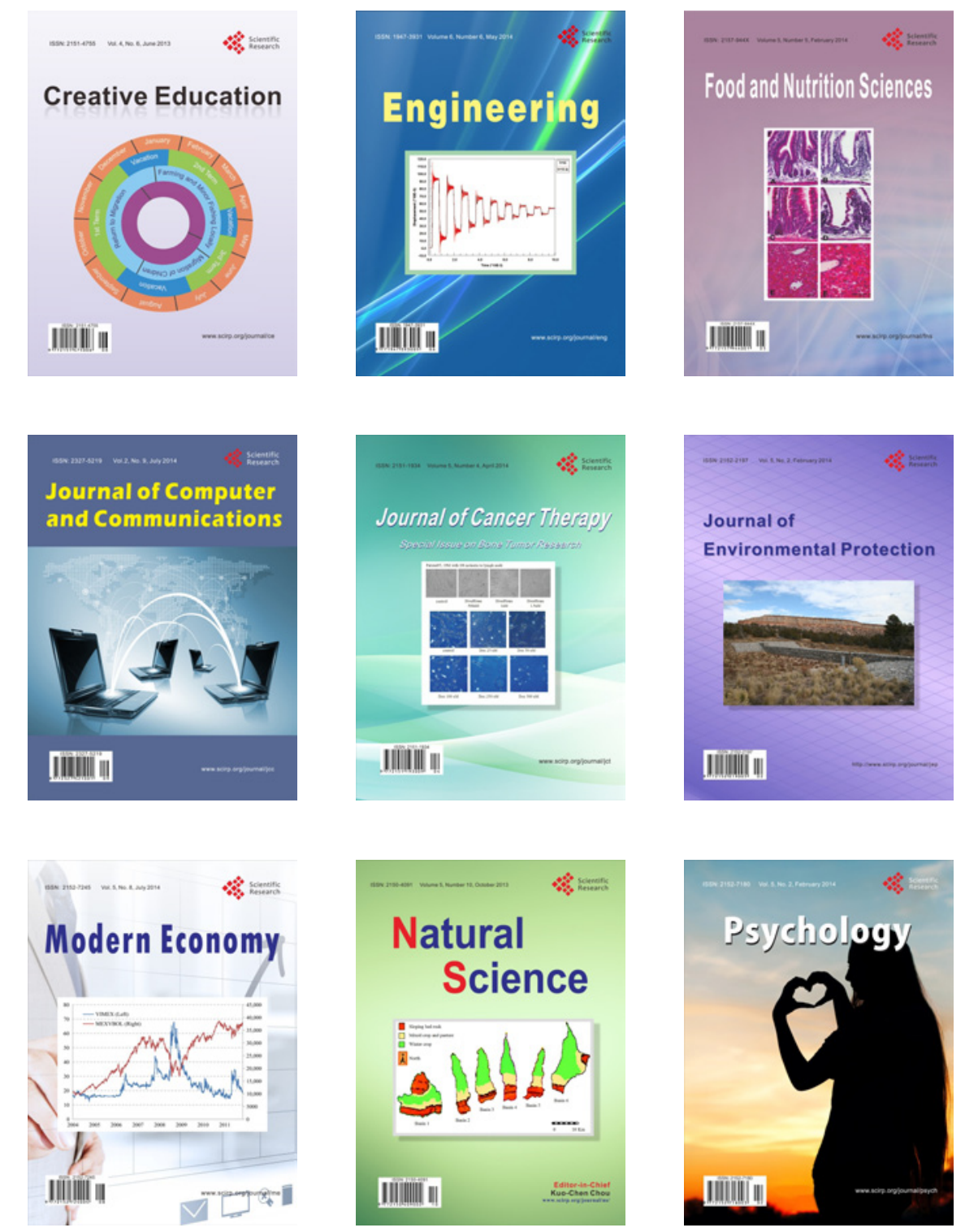